\title{
LETTER
}

\section{Operational sex ratio, sexual conflict and the intensity of sexual selection}

Patrick S. Fitze ${ }^{1,2 *}$ and JeanFrançois Le Galliard ${ }^{3}$

${ }^{1}$ Museo Nacional de Ciencias Naturales (MNCN-CSIC),

Department of Biodiversity and Evolutionary Biology, C/ José Gutiérrez Abascal 2, Madrid 28006, Spain ${ }^{2}$ Instituto Pirenaico de Ecología (IPE-CSIC), Avenida Regimiento de Galicia s/n, 22700 Jaca

(Huesca), Spain

${ }^{3}$ Laboratoire Fonctionnement et Evolution des Systèmes

Ecologiques, CNRS UMR 7625,

Université Pierre et Marie Curie, 7 Quai St Bernard, 75005 Paris, France *Correspondence: E-mail: patrick.fitze@mncn.csic.es

\begin{abstract}
Modern sexual selection theory indicates that reproductive costs rather than the operational sex ratio predict the intensity of sexual selection. We investigated sexual selection in the polygynandrous common lizard Lacerta vivipara. This species shows male aggression, causing high mating costs for females when adult sex ratios (ASR) are malebiased. We manipulated ASR in 12 experimental populations and quantified the intensity of sexual selection based on the relationship between reproductive success and body size. In sharp contrast to classical sexual selection theory predictions, positive directional sexual selection on male size was stronger and positive directional selection on female size weaker in female-biased populations than in male-biased populations. Thus, consistent with modern theory, directional sexual selection on male size was weaker in populations with higher female mating costs. This suggests that the costs of breeding, but not the operational sex ratio, correctly predicted the strength of sexual selection.
\end{abstract}

\section{Keywords}

Adult sex ratio, Lacerta vivipara, operational sex ratio, reproductive costs, sexual conflict, strength of sexual selection.

Ecology Letters (2008) 11: 432-439

\section{INTRODUCTION}

Sexual selection results from competition between individuals of one sex for access to gametes of the opposite sex, which leads to a non-random distribution of reproductive success among individuals (Darwin 1871). The investigation of factors affecting the strength of sexual selection is important for understanding sex roles (Andersson 1994), interspecific variation in sexual dimorphism (Møller 1994), and the evolutionary dynamics of sex-specific morphological and behavioural traits (Lande 1980). The most prevalent sexual selection theory predicts that sex roles in reproductive behaviour depend on the operational sex ratio (OSR), defined as the ratio of males to females ready to mate in a population at a given time (Emlen \& Oring 1977). This theory is based on the fact that the OSR is a major predictor of the level of mate competition (Kvarnemo \& Ahnesjo 1996) and, to some extent, of mate choice (Kokko \& Monaghan 2001). The operational sex ratio (OSR) is primarily determined by the adult sex ratio (ASR) and the potential reproductive rate (PRR), i.e. the relative time spent in reproductive vs. non-reproductive activity (Clutton-Brock \& Vincent 1991; Parker \& Simmons 1996). Therefore, the intensity of sexual selection should be stronger in the more common sex and the sex with the higher PRR (Bjork \& Pitnick 2006); this has been shown for bush crickets (Simmons 1992) and sand gobies (Kvarnemo 1996).

However, this straightforward relationship between the OSR, sex roles, and the intensity of sexual selection is inconsistent with other models of mate competition and mate choice proposing complex interactions between the OSR, parental investment and life history (Kokko \& Monaghan 2001; Kokko \& Johnstone 2002). Recent evolutionary models suggest that the sign and magnitude of the effect of OSR on the intensity of sexual selection depend on components of the mating system, such as mortality costs (Kokko \& Monaghan 2001; Kokko \& Johnstone 2002; Simmons \& Kvarnemo 2006) - during the time when an individual is not capable of mating (i.e. "time out', Clutton-Brock \& Parker 1992) and during the time when an individual is capable of mating (i.e. 'time in', Clutton-Brock \& Parker 1992) - alternative mating strategies (Mills \& Reynolds 2003), the prevalence of coercive mechanisms of sexual selection (Head \& Brooks 2006; Simmons \& Kvarnemo 2006), or mate encounter rates (Kokko \& Monaghan 2001; Kokko \& Johnstone 2002). We 
investigated the effects of OSR on the strength of the sexual selection in the common lizard (Lacerta vivipara Jacquin 1787), whose mating system is characterized by high polygynandry (Fitze et al. 2005), significant male aggression during mating (Le Galliard et al. 2005), and higher mating costs (mortality during time in) for females than for males when ASRs are biased towards males (Le Galliard et al. 2005).

The common lizard breeds annually, has an ovoviviparous reproductive system without parental care, and exhibits a polygynandrous mating system (Laloi et al. 2004). Males and females can reach sexual maturity in their first spring and have overlapping home ranges (Le Galliard et al. 2005). Adult males emerge from hibernation on average 1 month before the females and become sexually active after their first spring molt (Bauwens et al. 1989). Females emerge asynchronously, mate readily after attainment of sexual maturity, and can be receptive during a period of 1-2 weeks (Bauwens \& Verheyen 1985). The PRR of males and females is difficult to quantify in many species, including the common lizard (Parker \& Simmons 1996). However, experimental manipulation of the ASR within the range of temporal and spatial variability observed in the wild should induce predictable changes in the OSR (Kvarnemo 1996).

We subjected 12 experimental populations - six of which were male-biased and six female-biased - to ASR manipulation and microsatellite-based paternity analysis, including all offspring. Quantification of the strength of sexual selection acting on body size in male and female lizards was based on the relationship between reproductive success (i.e. number of progeny) and body size. Body size, assessed by the lizard's snout-vent length (SVL), is a critical determinant of life history variation in many taxa (Andersson 1994). In female common lizards, body size affects clutch size as well as the timing of reproductive events (Bauwens \& Verheyen 1985) and female mate choice (Richard et al. 2005). In male lizards, body size affects dominance status and endurance capacity, and therefore the ability to search for females and assure mating (Richard et al. 2005). We decomposed reproductive success into four multiplicative fitness components - mating success (number of mates), number of offspring per mate, hatching success and offspring survival until maturity (Clutton-Brock 1988) and quantified the relationship between these components and body size to estimate the strength of sexual selection acting on body size.

\section{MATERIALS AND METHODS}

\section{Experimental manipulation of the ASR}

Twelve experimental lizard populations $(10 \times 10 \mathrm{~m})$ enclosed by plastic walls were established in July 2002 at the research station at Foljuif (Seine et Marne, France). Each enclosure contained a patch of natural vegetation and two water ponds, which provided natural food and water for the lizards. The enclosures also contained four stone piles for use as basking sites and shelters (for more technical details, see Lecomte \& Clobert 1996). Plastic walls prevented lizards from escaping from the enclosures and 18 adult (over 1 year old), 12 yearling (1 year old), and 42-45 newborn juvenile common lizards were released into each of the enclosures at the start of the experiment. In six of the 12 populations, we biased the ASR towards males (MB); in the other six populations the ASR was biased towards females (FB). Fourteen adult males and four adult females were released in MB populations, and four adult males and fourteen adult females in FB populations. The ASR, defined as the number of adult males per number of adult individuals, was 0.78 in $\mathrm{MB}$ and 0.22 in $\mathrm{FB}$ populations. The sex ratio of the $\mathrm{FB}$ populations corresponded to the average ASR of similarly sized patches in a natural population of the native mountainous range in the Cévennes, Southern France (average ASR $=0.22 \pm 0.21 \mathrm{SD}, 22$ patches monitored over 13 years, data provided by M. Massot). The sex ratio of the MB populations corresponded to the largest sex ratios in the same population (see Appendix S1 for further details on the demographic structure of experimental populations).

\section{Assessment of reproductive characteristics}

At the end of the mating season, during late May 2003, all live lizards were captured within two consecutive days. The sex ratio of the populations at the end of mating season was still different between treatments (MB populations: $23.7 \pm 3.2 \mathrm{SE}$ males, $5.7 \pm 0.8 \mathrm{SE}$ females, $\mathrm{SR}=0.80 \pm 0.03 \mathrm{SE}, \mathrm{FB}$ populations: $15.8 \pm 2.5 \mathrm{SE}$ males, $20.7 \pm 2.1$ SE females, SR $=0.40 \pm 0.03$ SE; $\left.N=12, \quad F_{1,10}=113.24, P<0.001\right)$. Lizards were not captured during the mating season since this may have disturbed mate choice and mate competition, and thus would have affected the estimates of the intensity of sexual selection. Consequently, the exact numbers of males and females during the mating season is unknown. However, male survival was high in spring and was not affected by ASR manipulation (Le Galliard et al. 2005). Thus, the number of males observed corresponds closely to the number of males present during mating in both treatments.

After capture, snout-vent length (SVL) of each lizard was measured to the closest millimetre using a transparent ruler. Captured females were kept in the laboratory in individual terrariums under standardized conditions (water, heat and light regimes standardized according to Le Galliard et al. 
2003) and were fed with moth larvae every fourth day (Pyralis sp.). After each female laid its eggs, the terrarium was carefully searched for hatchlings and unhatched eggs. Hatching offspring were released after 2 days, into eight initially empty enclosures similar to those used for the ASR experiment. We released the same number of families in each population and made sure that the proportion of families originating from $\mathrm{MB}$ and $\mathrm{FB}$ populations was the same for each population (see Appendix S2 for detailed population structure and release protocol; Cote et al. 2007; Le Galliard et al. 2007). All surviving juveniles were recaptured approximately 11 months after hatching, in June 2004. This protocol allowed the longer-term effects of the 2002/2003 ASR on offspring viability selection to be quantified independently of the 2003/2004 ASR.

\section{Genetic protocol and assessment of paternity}

We collected a tissue sample of each individual before release, and of each hatchling and unhatched egg in 2003. Females laid a total of $753 \mathrm{eggs}, 687$ of which hatched. All tissues were stored in $60 \%$ ethanol. DNA of all tissue samples was extracted using Perfect gDNA Blood Mini Isolation kit for animal blood (Eppendorf, Hamburg, Germany). We used five to six highly polymorphic microsatellite DNA loci $(\mathrm{Lv}-3-19$, Lv-4-72, Lv-4-alpha, Lv-4-X, Lv-4-115 and Lv-2-145, Boudjemadi et al. 1999) to identify fathers. The methods used for DNA extraction, polymerase chain reaction (PCR), and determination of allelic size were previously described (Laloi et al. 2004). DNA of all but 12 unhatched eggs could be extracted. Thus, seven unhatched eggs of four different mothers originating from FB populations and five eggs of three different mothers originating from MB populations could not be included in the analyses. We carried out assignment tests using Cervus 2.0 (Marshall et al. 1998), to identify the genetic father for each hatchling and unhatched egg (Fitze et al. 2005).

\section{Intensity of sexual selection on body size}

Some, but not all, common lizards reproduce in their first spring (Boudjemadi et al. 1999). Immature female lizards are easily identified by absence of egg production, but it is not easy to distinguish mature males that did not father offspring due to male-male competition or female choice from sexually immature males unable to produce viable sperm. However, male size may indicate attainment of sexual maturity in lizards (Olsson \& Madsen 1996). Male size in our populations ranged from 44 to $65 \mathrm{~mm}$ $(N=225)$; the smallest male fertilizing eggs had an SVL of $50 \mathrm{~mm}$. There were only 12 potentially immature males $(5.3 \%)$ with a smaller SVL. Seven males lived in $\mathrm{MB}$ and five in $\mathrm{FB}$ populations, showing that they were evenly distributed among enclosures subjected to different ASR manipulations. Thus, we chose a conservative approach and included these males in the sexual selection analysis.

We quantified the strength of sexual selection in male and female lizards based on the relationship between reproductive success (number of progeny surviving until the age of 1 year) and body size (SVL). We decomposed reproductive success $W$ for males and females into four multiplicative fitness components, as follows:

$W=N_{\text {mate partners }} \times \phi_{N \text { eggs } / \text { mate partner }} \times H S \times \Phi_{\text {juvenile }}$

where $N_{\text {mate partners }}$ corresponds to the number of genetically determined mates, $\phi_{N \text { eggs } / \text { mate partner }}$ corresponds to the mean number of eggs per mate, $H S$ to the hatching success (i.e. the probability that an offspring hatched successfully from an egg), and $\Phi_{\text {juvenile survial }}$ is the survival rate from hatching to 1 year of age. Our analysis thus incorporates effects of body size on pre-copulatory sexual selection through mate competition and mate choice, as well as postcopulatory sexual selection through sperm choice, selective abortion of embryos or selective investment into offspring, and viability selection through juvenile survival (Andersson 1994; Eberhard 1996). We only included individuals that were alive during the mating season and our analysis of reproductive success was therefore not affected by natural selection through differential mortality of males and females prior to mating.

Variations in age may have a greater effect on mating tactics than body size; in such cases, the potential effect of body size on sexual selection would be confounded by age (Richard et al. 2005). We used long-term data to model the functional relationship between SVL and age in the common lizard (see Appendix S3 and S4 for data collection and analyses). On the basis of this analysis (see below), we calculated the standardized selection gradients for each population and each multiplicative fitness component, using a multiple regression with SVL and age as covariates (Lande \& Arnold 1983). To elucidate the selection mechanisms, we investigated whether the standardized fitness components were correlated, using simple regressions, and estimated the importance of each multiplicative fitness component for reproductive success, using a multivariate regression with the standardized reproductive success $(W)$ as a dependent variable and the standardized fitness components as covariates (Conner et al. 1996). We performed one-way ANOVA or Wilcoxon-signed ranks tests to determine whether the standardized selection gradients were significantly different between sex ratio manipulations. The quadratic selection gradients testing for stabilizing or disruptive selection were not significant. Hence, only the results of linear selection gradients $(\beta)$ are reported here. 


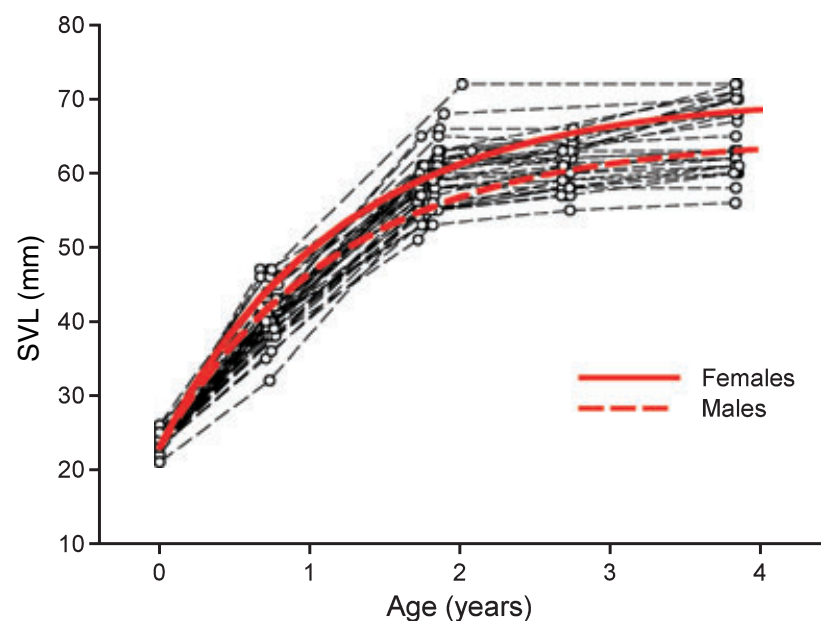

Figure 1 Body size growth trajectories of male and female common lizards. Body size growth trajectories of male and female common lizards from, birth to the age of 4 years, measured in experimental populations similar to, and from the same meadow as, those used for the ASR experiment (see Appendix S3 for further details). Individual growth curves for 42 lizards monitored until the age of 3 or 4 years (dashed black lines) are shown, with sex-specific estimates of the best fitting nonlinear growth model (red lines). The van Bertalanffy growth model included individual variation in asymptotic body size $\left(\chi^{2}=10.26, P=0.001\right)$ and exponential growth rate $\left(\chi^{2}=6.33, P=0.01\right)$, as well as sex differences in asymptotic body size $\left(F_{1,167}=28.54, P<0.0001\right)$. The predicted asymptotic body size was $70.3 \mathrm{~mm}(68.5,72.1) 95 \%$ CI for females and $64.8 \mathrm{~mm}(63.2,66.2)$ for males. See Appendix S3 and S4 for details.

\section{RESULTS}

The relationship between body size and age - ranging from 1 to 4 years - for common lizards was best described by a nonlinear growth model (Fig. 1). Females grew to a larger asymptotic body size than males (Fig. 1). Although common lizards show indeterminate growth, female and male lizards reached $86 \%$ and $85 \%$ of their asymptotic body size by the age of 2 years. Body size ranks of individual lizards remained constant after 2 years of age $\left(\chi^{2}=42.71\right.$, $P<0.001$, repeatability of individual body size: $r=0.65)$, but not from 1 year of age $\left(\chi^{2}<0.001, P>0.99\right.$, $r<0.001)$; this suggests the existence of compensatory growth early in life. The combination of fast growth early in life with significant and predictable individual variation in body size among adults enabled us to assess the combined effects of age and size on sexual selection without the complication of strong colinearity between age and body size (Quinn \& Keough 2002).

Current theory suggests that the strength of sexual selection in each sex is accurately predicted by the OSR, which is determined by the ASR (Emlen \& Oring 1977). It is generally assumed that the limiting sex is more selective in mate choice, thereby determining the strength of sexual selection in the predominant sex, which invests more in competition (Kvarnemo \& Ahnesjo 1996). Thus, sexual selection should be stronger for males in $\mathrm{MB}$ than in $\mathrm{FB}$ populations, whereas sexual selection in females should be stronger in FB than in MB populations. The number of mates was under strongest selection in our study; this is consistent with Bateman's principle of stronger selection for access to mates than for other components of sexual selection (Shuster \& Wade 2003; Fig. 2). On average, there was a positive correlation between the number of mates and body size in both sexes, indicating positive, directional sexual selection acting on body size. The sign of the effect of ASR manipulation on this gradient of sexual selection clearly opposes predictions based on the prevalent sexual selection theory (Emlen \& Oring 1977). The intensity of sexual selection acting on male body size, measured by the number of mates, was 4.4 times higher in populations where males were rare (FB: $\beta=0.518 \pm 0.03 \mathrm{SE}$ ) than in populations where males were abundant (MB: $\beta=0.117 \pm 0.04 \mathrm{SE} ; F_{1,10}=9.614, P=0.011$, Fig. $\left.2 \mathrm{a}\right)$. As for males, female body size was under stronger selection when females were rare. The intensity of sexual selection on female body size, as assessed from the number of male partners, was three times higher in $\mathrm{MB}(\beta=1.345 \pm 0.14$ $\mathrm{SE})$ compared with $\mathrm{FB}$ populations $(\beta=0.454 \pm 0.04 \mathrm{SE}$; Wilcoxon $W(\mathrm{WW})=0, P=0.008$, Fig. 2b). Age was not a good predictor of sexual selection (all $P>0.1$ ). Furthermore, there were no significant effects of ASR manipulation on the correlations between age (all $P>0.05$ ) or body size and the number of eggs per mate (males: $\beta_{\mathrm{FB}}=$ $0.017 \pm 0.08 \mathrm{SE} ; \beta_{\mathrm{MB}}=0.431 \pm 0.17 \mathrm{SE}, F_{1,10}=0.815$, $P=0.388 ; \quad$ females: $\quad \beta_{\mathrm{FB}}=0.496 \pm 0.08$ SE; $\beta_{\mathrm{MB}}=$ $\left.-0.067 \pm 0.14 \mathrm{SE}, F_{1,8}=3.10, P=0.116\right)$, hatching success (males: $\beta_{\mathrm{FB}}=0.397 \pm 0.10 \mathrm{SE} ; \beta_{\mathrm{MB}}=0.291 \pm 0.07 \mathrm{SE}$, $F_{1,9}=0.126, P=0.731$; females: $\beta_{\mathrm{FB}}=0.132 \pm 0.12 \mathrm{SE}$; $\beta_{\mathrm{MB}}=2.099 \pm 0.53 \mathrm{SE}, \mathrm{WW}=5, P=0.166$ ), or juvenile survival (males: $\beta_{\mathrm{FB}}=0.156 \pm 0.13 \mathrm{SE} ; \beta_{\mathrm{MB}}=0.128 \pm$ 0.596 SE, $\quad F_{1,7}=0.001, \quad P=0.973$; females: $\beta_{\mathrm{FB}}=$ $-0.041 \pm 0.10 \mathrm{SE} ; \beta_{\mathrm{MB}}=-6.768 \pm 2.66 \mathrm{SE}, \mathrm{WW}=12$, $P=0.067$, Fig. 2).

\section{DISCUSSION}

Until recently, is has been widely accepted that the strength of sexual selection is mainly determined by the operational sex ratio and that sexual selection is stronger in the abundant sex (Emlen \& Oring 1977). However, the findings from a number of empirical studies are not consistent with these predictions. For example, Van Dongen et al. (1998) found that male, but not female, winter moths (Operopthera brumata) were choosing mates, despite a heavily male-biased 
(a) Males

SVL

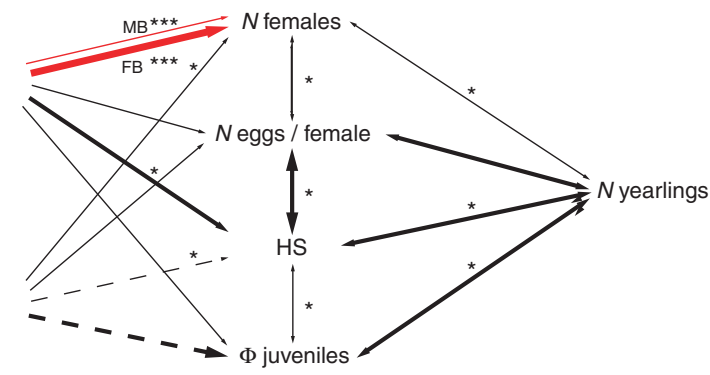

(b) Females
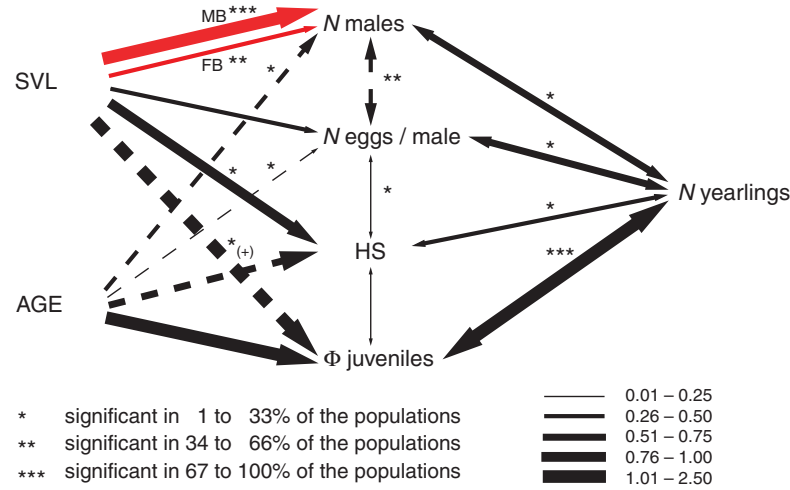

Figure 2 Path diagrams of selection acting on fitness. Path diagrams for selection acting on the four multiplicative fitness components of selection in male (a) and female (b) common lizards. Each arrow represents the mean standardized selection gradient for the twelve experimental populations (six MB and six FB populations). Significant differences in the strength of selection between $\mathrm{MB}$ and $\mathrm{FB}$ populations are represented by separate arrows. Correlations are depicted as double-headed arrows, and causal relationships as single-headed arrows. Dashed arrows denote negative coefficients. Arrow width is proportional to the standardized coefficient (see scale). Stars indicate the significance level of the respective relationships. HS refers to hatching success and $\Phi$ refers to juvenile survival (see eqn 1). Red arrows depict significant differences in the strength of selection between female-biased (FB) and male-biased (MB) populations (for further details see Appendix S5). For males, the intensity of sexual selection on the number of mates was significantly higher in $\mathrm{FB}$ than in $\mathrm{MB}$ populations $\left(F_{1,10}=9.614, P=0.011\right)$; for females, the intensity of sexual selection on the number of mates was significantly higher in $\mathrm{MB}$ populations (WW $=0, P=0.008$ ). For males in $\mathrm{FB}$ populations, reproductive success was mainly determined by the number of mates $\left(R^{2}=0.206 \pm 0.100 \mathrm{SE}\right)$, whereas the number of eggs fertilized per female $\left(R^{2}=0.061 \pm 0.004 \mathrm{SE}\right)$, the hatching success $\left(R^{2}=0.094 \pm 0.083 \mathrm{SE}\right)$, and the juvenile survival $\left(R^{2}=0.053 \pm 0.019 \mathrm{SE}\right)$ accounted for less of the variance in total fitness. For females in MB populations, reproductive success was mainly determined by juvenile survival $\left(R^{2}=0.491 \pm 0.117\right.$ $\mathrm{SE})$, and to a lesser extent by the number of mates $\left(R^{2}=0.065 \pm\right.$ $0.021 \mathrm{SE})$, the number of eggs per mate $\left(R^{2}=0.044 \pm 0.014 \mathrm{SE}\right)$, and hatching success $\left(R^{2}=0.077 \pm 0.066 \mathrm{SE}\right)$.
OSR. Recently, Kokko and Monaghan (2001) and Kokko and Johnstone (2002) designed an evolutionary model showing that the OSR may be a good predictor of the intensity of sexual selection in some circumstances, but that parental investment and mating costs are the primary determinants of sex roles. The factors promoting 'choosiness' in one sex are high sex-specific mortality costs of breeding (mortality during 'time out' and relative duration of 'time out'), high sex-specific mate-encounter rate, low sexspecific mortality during 'time in', and highly variable quality of the opposite sex (Kokko \& Johnstone 2002).

In this study, we manipulated the OSR in replicated populations and tested the effect of MB OSR on the strength of sexual selection acting on body size. We assessed reproductive success (the number of genetic offspring) in adults surviving until the mating season and quantified the intensity of sexual selection with standardized selection gradients - a common practice for measuring the intensity of sexual selection (Shuster \& Wade 2003). This approach excludes differential survival selection between individuals subjected to different ASR treatments and thus allows exclusive quantification of sexual selection intensity. Furthermore, we decomposed reproductive success into four multiplicative terms: the number of genetic mates, the number of genetic offspring per mate, the hatching success of the genetic offspring, and the juvenile survival of the genetic hatchlings. Hence, we were able to investigate the effects of OSR variation on pre-copulatory and postcopulatory components of sexual selection in far more detail than in the previous studies.

Our findings are consistent with predictions based on the sexual selection models recently proposed by Kokko and Monaghan (2001) and Kokko and Johnstone (2002). We previously reported that mating costs for females during 'time in' were higher in $\mathrm{MB}$ than in FB populations due to sexual harassment by males (Le Galliard et al. 2005). Further, females of MB populations produced fewer offspring during the ASR manipulation than females of FB populations (Le Galliard et al. 2005) and had higher survival rates in the year following the ASR manipulation (Le Galliard et al. 2007). Thus, breeding costs of females of $\mathrm{MB}$ populations seem to be lower than those of FB populations. Mate encounter rates and variation in mate quality did not differ between enclosures subjected to different ASR manipulations (Fitze et al. 2005). Similarly, variation in mortality during 'time out' did not differ between females or males subjected to different ASR perturbations (Le Galliard et al. 2005). According to the recent sexual selection models (Kokko \& Monaghan 2001; Kokko \& Johnstone 2002), both higher costs of mating (higher mortality during 'time in') and lower costs of breeding for females of MB populations should reduce 
female choosiness. Consequently, there should be weaker sexual selection - in terms of access to mates - on male body size. Indeed, we found that the strength of sexual selection on male SVL was weaker in MB than in FB populations. The classic models of sexual selection predict that the competition among males for access to females should be higher in $\mathrm{MB}$ than in FB populations (Emlen \& Oring 1977). However, our findings are clearly inconsistent with these models: the ASR-induced shift in the strength of sexual selection in males was opposite to that predicted by classical models. Furthermore, ASR manipulation had no effect on male survival (Le Galliard et al. 2005) and on survival selection acting on male body size between July 2002 and May 2003 (see Appendix S6); this indicates that the strength of intrasexual competition between males was similar between enclosures subjected to different sex ratio manipulations.

The recent sexual selection models also predict that increased selection in one sex may decrease selection in the other sex (Kokko \& Monaghan 2001; Kokko \& Johnstone 2002). Again, our experimental results are consistent with this prediction: the intensity of selection acting on female body size, as assessed by the number of mates, was higher in $\mathrm{MB}$ populations than in FB populations. Increased selection on female body size in MB populations may potentially result from size-dependent costs of increased male aggression for females of MB populations (Head \& Brooks 2006). Larger females from MB but not from FB populations showed more mating scars than small females, and larger females may be less sensitive to increased male aggression than smaller females (Fitze et al. 2005; Le Galliard et al. 2005). Differences in selection on female body size may also arise due to male mate choice. Yet, given that male mate choice has been rarely reported in reptiles (Orrell \& Jenssen 2002), male mate choice is less likely to be responsible for stronger selection on females of MB populations.

The observed pattern summarized for females in Figure 2 may not necessarily imply sexual selection if the number of mate partners is not causally linked with reproductive success (Shuster \& Wade 2003). For example, larger females might be more harassed by males and consequently they may have mated more often, but female body size may independently determine clutch size, hence reproductive success. Alternatively, females may benefit from multiple mating through increased ovulation (Fitze et al. 2005; Eizaguirre et al. 2007) and thus the number of mate partners may be causally linked with reproductive success. We tested this hypothesis by analysing the relationship between clutch size, female size and multiple mating, assessed by the number of mating scars - a good proxy for the number of mating attempts (Fitze et al. 2005). We found that clutch size, independent of body size, increased with the number of mating scars, and that the increase did not significantly differ between ASR treatments, i.e. no significant interaction between ASR and number of mating scars existed [ $N$ mating scars: $F_{1,143}=12.99, P<0.001$, estimate: $0.211 \pm 0.06 \mathrm{SE}$; SVL $F_{1,143}=240.65, P<0.001$, estimate: $0.204 \pm 0.01 \mathrm{SE}$; ASR effect: $\quad F_{1,10}=12.62, \quad P=0.005$, estimate(FB): $0.441 \pm 0.12 \mathrm{SE}$; interaction: $\mathrm{ASR} \times N$ mating scars $F_{1,142}=0.105, P=0.746$, estimate: $\left.0.020 \pm 0.06 \mathrm{SE}\right]$. This suggests that multiple mating, independent of body size, let to increased clutch size, indicating that the relationship between multiple mating and reproductive success could be causal in female common lizards (Fitze et al. 2005; Eizaguirre et al. 2007) and indicating that sexual selection may also be responsible for the observed patterns in females. Future studies should further clarify the link between multiple mating and female fecundity and the importance of sexual selection through multiple mating in females.

Our study demonstrates a relationship between OSR and the intensity of sexual selection opposite to that predicted by classical sexual selection theory (Emlen \& Oring 1977). However, our findings are consistent with recent sexual selection models (Kokko \& Monaghan 2001; Kokko \& Johnstone 2002) that emphasize the importance of measuring both mating and breeding costs to understand sexual selection. Kokko \& Monaghan (2001) suggested that studies of sexual selection in species with flexible mating systems and life histories may be useful for elucidating how OSR and life history predict the intensity of sexual selection. In a recent experiment, Simmons \& Kvarnemo (2006) showed that in the Australian tettigoniid, Kawanaphila nartee, PRR, which were determined by the time required for a breeding attempt, predicted sexual selection better than breeding costs. This demonstrates that, in contrast to the common lizard, variation in mating and breeding mortality (cost of breeding) is not an important determinant of sexual selection in $K$. nartee. Kokko \& Monaghan (2001) and Kokko \& Johnstone (2002) stated that if they were to simplify sexual selection to a single parameter that best predicts a mating system', it would be the mortality costs of breeding. However, this simplification would lead to incorrect predictions in both our and Simmons \& Kvarnemo's (2006) studies; these studies thus demonstrate that the complexity of sexual selection cannot be reduced to a single parameter.

Our findings have important implications for our understanding of the ecological context of sexual selection and the long-term evolution of sexually dimorphic characters, such as body size. They highlight that the balance between mate choice, mate competition and sexual conflict may determine how biased sex ratios influence the strength of sexual selection on body size. Intersexual conflicts may therefore affect the evolution of sexual size dimorphism (Szekely et al. 2004), including growth and reproductive tactics (Ramm 2007), and they may significantly determine 
the speciation (Gavrilets \& Waxman 2002; Masta \& Maddison 2002; Martin \& Hosken 2003) of sexually reproducing organisms.

\section{ACKNOWLEDGEMENTS}

We thank J. Cote, D. Mersch, and M.-L. Jarzat for assistance during population monitoring. Our research was funded by the French Ministry of Education and Research (ACI 'Jeunes Chercheurs 2001'), the French Ministry of Environment (ACI 'Invasions Biologiques'), the Federal Office of Education and Science (BBW Grant 01.0254 to P.S.F), and the Spanish Ministry of Education and Science (CGL2005-01187).

\section{REFERENCES}

Andersson, M. (1994). Sexual Selection. Princeton University Press, Princeton.

Bauwens, D. \& Verheyen, R.F. (1985). The timing of reproduction in the lizard Lacerta vivipara differences between individual females. J. Herpetol., 19, 353-364.

Bauwens, D., Vandamme, R. \& Verheyen, R.F. (1989). Synchronization of spring molting with the onset of mating-behavior in male lizards, Lacerta vivipara. J. Herpetol., 23, 89-91.

Bjork, A. \& Pitnick, S. (2006). Intensity of sexual selection along the anisogamy-isogamy continuum. Nature, 441, 742-745.

Boudjemadi, K., Martin, O., Simon, J.C. \& Estoup, A. (1999). Development and cross-species comparison of microsatellite markers in two lizard species, Lacerta vivipara and Podarcis muralis. Mol. Ecol., 8, 518-520.

Clutton-Brock, T.H. (1988). Reproductive Success - Studies of Individual Variation in Contrasting Breeding Systems. University of Chicago Press, Chicago.

Clutton-Brock, T.H. \& Parker, G.A. (1992). Potential reproductive rates and the operation of sexual selection. Q. Rev. Biol., 67, 437456.

Clutton-Brock, T.H. \& Vincent, A.C.J. (1991). Sexual selection and the potential reproductive rates of males and females. Nature, $351,58-60$.

Conner, J.K., Rush, S. \& Jennetten, P. (1996). Measurements of natural selection on floral traits in wild radish (Raphanus raphanistrum) .1. Selection through lifetime female fitness. Evolution, 50, 1127-1136.

Cote, J., Clobert, J. \& Fitze, P.S. (2007). Mother - offspring competition promotes colonization success. Proc. Natl Acad. Sci. USA, 104, 9703-9708.

Darwin, C. (1871). The Descent of Man, and Selection in Relation to Sex. Appleton, New York.

Eberhard, W.G. (1996). Female Control: Sexual Selection by Cryptic Female Choice. Princeton University Press, Princeton, NJ.

Eizaguirre, C., Laloi, D., Massot, M., Richard, M., Federici, P. \& Clobert, J. (2007). Condition dependence of reproductive strategy and the benefits of polyandry in a viviparous lizard. Proc. $R$. Soc. B Biol. Sci., 274, 425-430.

Emlen, D.J. \& Oring, L.W. (1977). Ecology, sexual selection, and the evolution of mating systems. Science, 197, 215-223.

Fitze, P.S., Le Galliard, J.-F., Federici, P., Richard, M. \& Clobert, J. (2005). Conflict over multiple partner mating between males and females of polygynandrous common lizards. Evolution, 59, 2451 2459.

Gavrilets, S. \& Waxman, D. (2002). Sympatric speciation by sexual conflict. Proc. Natl Acad. Sci. USA, 99, 10533-10538.

Head, M.L. \& Brooks, R. (2006). Sexual coercion and the opportunity for sexual selection in guppies. Anim. Behav., 71, 515-522.

Kokko, H. \& Johnstone, R.A. (2002). Why is mutual mate choice not the norm? Operational sex ratios, sex roles and the evolution of sexually dimorphic and monomorphic signalling Philos. Trans. $R$ Soc. Lond. Ser. B Biol. Sci., 357, 319-330.

Kokko, H. \& Monaghan, P. (2001). Predicting the direction of sexual selection. Ecol. Lett., 4, 159-165.

Kvarnemo, C. (1996). Temperature affects operational sex ratio and intensity of male-male competition: Experimental study of sand gobies, Pomatoschistus minutus. Behav. Ecol., 7, 208-212.

Kvarnemo, C. \& Ahnesjo, I. (1996). The dynamics of operational sex ratios and competition for mates. Trends Ecol. Evol., 11, 404 408.

Laloi, D., Richard, M., Lecomte, J., Massot, M. \& Clobert, J. (2004). Multiple paternity in clutches of common lizard Lacerta vivipara: data from microsatellite markers. Mol. Ecol., 13, 719-723.

Lande, R. (1980). Sexual dimorphism, sexual selection, and adaptation in polygenic characters. Evolution, 34, 292-305.

Lande, R. \& Arnold, S.J. (1983). The measurement of selection on correlated characters. Evolution, 37, 1210-1226.

Le Galliard, J.-F., Ferrière, R. \& Clobert, J. (2003). Motheroffspring interactions affect natal dispersal in a lizard. Proc. $R$. Soc. Lond. B. Biol. Sci., 270, 1163-1169.

Le Galliard, J.-F., Fitze, P.S., Ferrière, R. \& Clobert, J. (2005). Sex ratio bias, male aggression, and population collapse in lizards. Proc. Natl Acad. Sci. USA, 102, 18231-18236.

Le Galliard, J.-F., Cote, J. \& Fitze, P.S. (2008). Lifetime and intergenerational fitness consequences of harmful male interactions for female lizards. Ecology, 89, 56-64.

Lecomte, J. \& Clobert, J. (1996). Dispersal and connectivity in populations of the common lizard Lacerta vivipara: An experimental approach. Acta Oecol. Int. J. Ecol., 17, 585-598.

Marshall, T.C., Slate, J., Kruuk, L.E.B. \& Pemberton, J.M. (1998). Statistical confidence for likelihood-based paternity inference in natural populations. Mol. Ecol., 7, 639-655.

Martin, O.Y. \& Hosken, D.J. (2003). The evolution of reproductive isolation through sexual conflict. Nature, 423, 979-982.

Masta, S.E. \& Maddison, W.P. (2002). Sexual selection driving diversification in jumping spiders. Proc. Natl Acad. Sci. USA, 99, 4442-4447.

Mills, S.C. \& Reynolds, J.D. (2003). Operational sex ratio and alternative reproductive behaviours in the European bitterling, Rhodeus sericeus. Behav. Ecol. Sociobiol., 54, 98-104.

Møller, A.P. (1994). Sexual Selection and the Barn Swallow. Oxford University Press, Oxford.

Olsson, M. \& Madsen, T. (1996). Costs of mating with infertile males selects for late emergence in female sand lizards (Lacerta agilis L).. Copeia, 2, 462-464.

Orrell, K.S. \& Jenssen, T.A. (2002). Male mate choice by the lizard Anolis carolinensis: a preference for novel females. Anim. Behav., 63, 1091-1102.

Parker, G.A. \& Simmons, L.W. (1996). Parental investment and the control of sexual selection: predicting the direction of sexual competition. Proc. $R$ Soc. Lond. Ser. B Biol. Sci., 263, 315-321. 
Quinn, G.P. \& Keough, M.J. (2002). Experimental Design and Data Analysis for Biologists. Cambridge University Press, Cambridge.

Ramm, S.A. (2007). Sexual selection and genital evolution in mammals: a phylogenetic analysis of baculum length. Am. Nat., 169, 360-369.

Richard, M., Lecomte, J., de Fraipont, M. \& Clobert, J. (2005). Agespecific mating strategies and reproductive senescence. Mol. Ecol., 14, 3147-3155.

Shuster, S.M. \& Wade, J.M. (2003). Mating Systems and Strategies. Princeton University Press, Princeton, NJ.

Simmons, L.W. (1992). Quantification of role reversal in relative parental investment in a bush cricket. Nature, 358, 61-63.

Simmons, L.W. \& Kvarnemo, C. (2006). Costs of breeding and their effects on the direction of sexual selection. Proc. $R$ Soc. Lond. Ser. B Biol. Sci., 273, 465-470.

Szekely, T., Freckleton, R.P. \& Reynolds, J.D. (2004). Sexual selection explains Rensch's rule of size dimorphism in shorebirds. Proc. Natl Acad. Sci. USA, 101, 12224-12227.

Van Dongen, S., Matthysen, E., Sprengers, E. \& Dhondt, A.A. (1998). Mate selection by male winter moths Operophtera brumata (Lepidoptera, Geometridae): adaptive male choice or female control? Behaviour, 135, 29-42.

\section{SUPPLEMENTARY MATERIAL}

The following supplementary material is available for this article:

Appendix S1 Detailed demographic structure of experimental populations.
Appendix S2 Demographic structure of experimental populations during the second year of the study.

Appendix S3 Long-term study of growth patterns.

Appendix S4 Statistical analysis of growth trajectories.

Appendix S5 Statistical analysis of the intensity of sexual selection.

Appendix S6 Statistical analysis of survival selection acting on male body size and body condition.

This material is available as part of the online article from: http://www.blackwell-synergy.com/doi/full/10.1111/ j.1461-0248.2008.01158.x.

Please note: Blackwell publishing are not responsible for the content or functionality of any supplementary materials supplied by the authors. Any queries (other than missing material) should be directed to the corresponding author for the article.

Editor, Ross Crozier

Manuscript received 22 November 2007

First decision made 19 December 2007

Manuscript accepted 5 January 2008 